Check for updates

Cite this: Chem. Sci., 2019, 10, 3307

๑ All publication charges for this article have been paid for by the Royal Society of Chemistry

Received 3rd January 2019

Accepted 29th January 2019

DOI: $10.1039 / \mathrm{c} 9 \mathrm{sc00032a}$

rsc.li/chemical-science

\section{Space-confined indicator displacement assay inside a metal-organic framework for fluorescence turn-on sensing $\dagger$}

\author{
Ning-Ning Yang, Li-Jiao Zhou, Peng Li, Qi Sui and En-Qing Gao (DD*
}

The indicator displacement assay (IDA) is for the first time performed within a metal-organic framework (MOF) to achieve ultrasensitive fluorescence turn-on sensing. A Zr(Iv) ion MOF (UiO-67-DQ-PsO) furnished with electron-deficient diquat units $\left(\mathrm{DQ}^{2+}\right.$, as the receptor) on the wall and electron-rich 1-pyrenesulfonate anions ( $\mathrm{PsO}^{-}$, as the fluorescent indicator) in the pores was prepared by postsynthetic anion exchange. The MOF is capable of sensing alkylamines owing to the competing $\mathrm{PsO}^{-}-\mathrm{DQ}^{2+}$ and alkylamine- $\mathrm{DQ}^{2+}$ charge-transfer interactions, the former interaction causing a fluorescence OFF state and the latter displacing $\mathrm{PsO}^{-}$to trigger its emission. Significant advantages have been demonstrated for the IDA inside the MOF. The turn-on assay exhibits much higher sensitivity and anti-interference than the turn-off sensing using the MOF without indicators (the sensitivity is enhanced by as much as six orders of magnitude to the subnanomolar level). The integration of both the receptor and indicator in the porous solid enables facile regeneration and recyclability of the IDA ensemble. Furthermore, we show that the confined space provided by the MOF significantly enhances the supramolecular interactions to make possible the IDA impossible in solution. This work not only demonstrates a novel conceptual approach to fabricate superior fluorescence turn-on sensors using porous materials but also has important implications for supramolecular chemistry in porous materials.

\section{Introduction}

Luminescence is a simple, rapid, and highly sensitive signal transduction method, so there has been an ever-escalating interest in the development of chemosensors using luminescent materials. Metal-organic frameworks (MOFs), a huge class of crystalline porous materials built of metal ions/clusters and organic linkers, provide superior platforms for fabricating luminescence sensing systems. ${ }^{1}$ The frameworks can be imparted with tunable luminescence through use of variable linkers, metal nodes, guests or inter-component cooperation. ${ }^{2}$ The porous matrices can preconcentrate analytes, show size/shape effects, and be modified with diverse functionalities through pre- and post-synthetic methods to provide or improve sensing performance. ${ }^{1,3}$ In particular, analyte-responsive receptors/reporters can be installed into the pores or on the surface through chemical bonds or supramolecular encapsulation. ${ }^{4}$ Furthermore, the confined space can allow for more efficient implementation of various sensing mechanisms through enhancing receptor-analyte interactions, ${ }^{5}$ promoting electron or energy transfer, ${ }^{6}$ and

Shanghai Key Laboratory of Green Chemistry and Chemical Processes, School of Chemistry and Molecular Engineering, East China Normal University, Shanghai 200062, P. R. China. E-mail: eqgao@chem.ecnu.edu.cn

$\dagger$ Electronic supplementary information (ESI) available: Supplementary graphics, Fig. S1-S13. See DOI: 10.1039/c9sc00032a facilitating exciplex formation. ${ }^{7}$ A great number of MOFs have been explored as luminescent sensors for a variety of species pertinent to environmental pollution, public safety or medical health. ${ }^{8}$ Most of them involve luminescence quenching (turn-off). More attractive but much more challenging is luminescence turnon sensing, where an analyte shows emission in a dark or weakly emissive state or causes an obvious shift in emission wavelength. ${ }^{9}$ The turn-on mode can have improved signal-to-noise ratios and anti-interference capability. However, turn-on sensing MOFs are still rare and most have some or other drawbacks, such as insufficiently weak original emission affecting sensitivity/ selectivity, irreversible reactions disenabling material reuse and on-line detection, poor water stability limiting applications under aqueous or humid conditions, and poor manipulability of the mechanism hampering systematic material optimization. The rational design of sensing systems that make full use of the advantages of MOFs and turn-on mechanisms is still challenging.

We propose a rational approach to fabricate turn-on sensors, utilizing the concept of indicator-displacement assays (IDAs) and the confined pore systems of MOFs. The IDA is a popular method to convert receptors into sensors making use of dynamic supramolecular interactions and has led to many sensing ensembles composed of various receptors and fluorescent or colorimetric indicators, targeting analytes of many classes. ${ }^{10}$ However, it has not yet been applied to MOFs. We propose that IDA can be performed within the pores of 
appropriate MOFs. Given the vast structural diversity and modifiability of MOFs, it should be possible to integrate both a receptor and fluorescent indicator in a MOF by framework functionalization and guest encapsulation. The receptor-indicator interaction could quench the emission from the indicator, giving an OFF state. The introduction of an analyte interacting with the receptor would separate the indicator from the receptor and thus evoke the fluorescence of the indicator. The crux for the turn-on sensing is the competition between the analyte and the indicator for interacting with the receptor. We envision that the host-guest interactions could be regulated or enhanced by the confined porous matrices resulting in high sensitivity and selectivity. In addition, the IDA inside a stable MOF should facilitate material regeneration and reuse (Scheme 1).

For a proof-of-concept study, we demonstrate the IDA of amines inside an electron-deficient MOF, which proceeds through competing donor-acceptor charge-transfer (CT) interactions. Amines are important substances having wide applications but are also notorious pollutants harmful to human health and the environment. ${ }^{11}$ An increasing number of MOFs have been reported to be luminescently responsive to amines, ${ }^{12}$ of which only a few show a turn-on response. ${ }^{13}$ A judicious strategy toward amine-sensing MOFs is to incorporate electron-deficient units, with which electron-rich amines can form CT complexes to induce photophysical changes. Thus, some MOFs bearing a naphthalenediimide or 4,4'-bipyridinium unit can sense amines in the turn-off mode, ${ }^{\mathbf{1 4}}$ and a dynamic coordination polymer shows a turn-on response to arylamines owing to emissive $\pi-\pi$ CT complexation. ${ }^{5 a}$ However, highly sensitive MOFs for sensing amines in aqueous media are still rare. Recently, water-stable ionic MOFs furnished with electrondeficient 2,2'-bipyridinium and exchangeable anions have been prepared through postsynthetic $\mathrm{N}$-alkylation of a 2,2'-bipyridylsupported $\mathrm{Zr}(\mathrm{Iv})$ MOF (UiO-67-bpy). ${ }^{15}$ It has been demonstrated that UiO-67-MQ ( $\mathrm{MQ}^{2+}=N, N^{\prime}$-dimethyl-2,2'-bipyridinium) can sense alkylamines through reversible color and fluorescence changes induced by amine-bipyridinium CT complexation (Scheme 1a). ${ }^{15 c}$ However, the fluorescence sensitivity is low owing to the weak fluorescence of the MOF and the turn-off mode. We envision that electron-deficient MOFs should be nice candidates

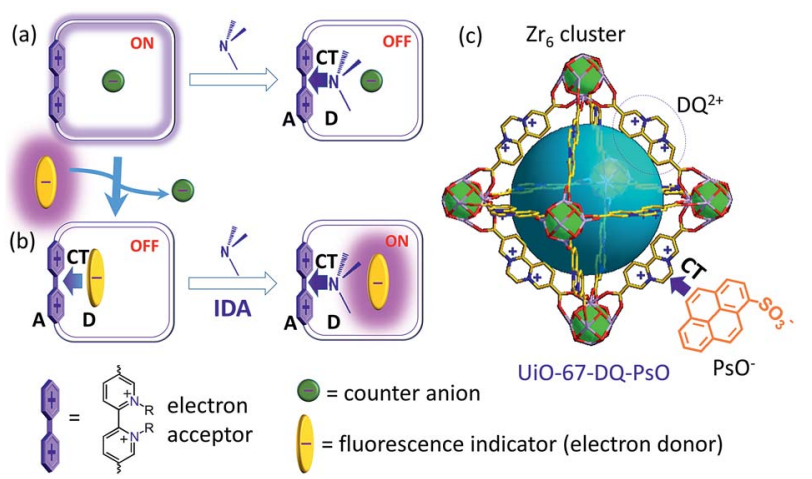

Scheme 1 (a) Fluorescence turn-off sensing of amine. (b) Turn-on sensing based on IDA. (c) The IDA in a MOF. CT = charge transfer, $A=$ acceptor, and $\mathrm{D}=$ donor. for turn-on IDAs through dynamic CT interactions. To implement this idea, an electron-rich and fluorescent anion (indicator) can be encapsulated into the MOF through anion exchange (Scheme 1b). The CT complexation between bipyridinium and the anion could quench the emission from the anion as well as that from the host. The amine-bipyridinium CT complexation could separate the anion from bipyridinium and thus the fluorescence of the anion could be turned on. With an appropriate anion, it should be possible to improve not only sensitivity but also selectivity. Here, we report the first example of the approach, using a MOF (UiO-67-DQ-PsO) integrated with diquat $\left(\mathrm{DQ}^{2+}\right)$ units as receptor sites and 1-pyrenesulfonate $\left(\mathrm{PsO}^{-}\right)$as the indicator (Scheme 1c). The sensing properties of the precursor MOF (UiO-67-DQ-TfO with $\mathrm{TfO}^{-}=$triflate) are also presented first for comparison. As will be shown, the turn-on IDA of alkylamines in water using UiO-67-DQ-PsO shows dramatically improved sensitivity and anti-interference capability. Other benefits of implementing IDA within a MOF and the interactions underlying the IDA mechanism will be discussed. Notably, the space confinement effect is critical in enabling the IDA.

\section{Results and discussion}

\section{Synthesis and characterization}

UiO-67-DQ-TfO was prepared by postsynthetic $N, N^{\prime}$-cycloalkylation of UiO-67-bpy with ethylene ditriflate (EDT) at room temperature, and UiO-67-DQ-PsO was obtained by anion exchange of UiO-67-DQ-TfO with sodium 1-pyrenesulfonate (NaPsO) in water. The reactions are confirmed by the IR spectra of the solids (Fig. S1 $\dagger$ ) and ${ }^{1} \mathrm{H}$ NMR spectra of the solutions obtained by digesting the solids in HF (aq.)/d6-DMSO. The success of $\mathrm{N}$-alkylation is indicated by the appearance of the characteristic IR bands of the ethylene group $\left(3086 \mathrm{~cm}^{-1}\right)$ and the triflate counteranion $\left(1280,1255,1170\right.$ and $\left.1030 \mathrm{~cm}^{-1}\right) \cdot{ }^{16}$ Anion exchange is indicated by the appearance of new multiple bands in the ranges of 1245-1160, 1090-1010, and 850$840 \mathrm{~cm}^{-1}$, which also appear in the spectra of NaPsO. ${ }^{1} \mathrm{H}$ NMR spectra show new peaks characteristic of the $\mathrm{DQ}^{2+}$ cationic moiety (marked with $\Delta$ ) after $\mathrm{N}$-alkylation and new peaks characteristic of the $\mathrm{PsO}^{-}$anion (marked with $\times$) after anion exchange (Fig. 1). The N-alkylation ratio and anion exchange ratio were calculated according to integral analyses of the NMR spectra. A maximum $\mathrm{N}$-alkylation ratio of about $65 \%$ can be achieved by prolonged reactions, ${ }^{15 d}$ but anion exchange of the highly N-alkylated MOF is incomplete (67\%, Fig. S2 $\dagger$ ), perhaps because the pore size of the MOF prevents encapsulation of more $\mathrm{PsO}^{-}$anions, which are much bulkier than $\mathrm{TfO}^{-}$. The incomplete anion exchange has an adverse effect on the sensing properties (see below). Complete anion exchange (the molar ratio of the $\mathrm{DQ}^{2+}$ cation to the $\mathrm{PsO}^{-}$anion is $1: 2$ as required by charge compensation) was achieved using UiO-67-DQ-TfO with a $\mathrm{N}$-alkylation ratio of $42 \%$ (Fig. 1), which was obtained by reducing the duration time of the $\mathrm{N}$-alkylation reaction. Hereafter unless otherwise specified, UiO-67-DQ-TfO refers to the MOF with the $42 \%$ modification ratio and UiO-67-DQ-PsO refers to the MOF after complete anion exchange. It is worth noting that the ${ }^{1} \mathrm{H}$ NMR peaks of DQ are obviously shifted 


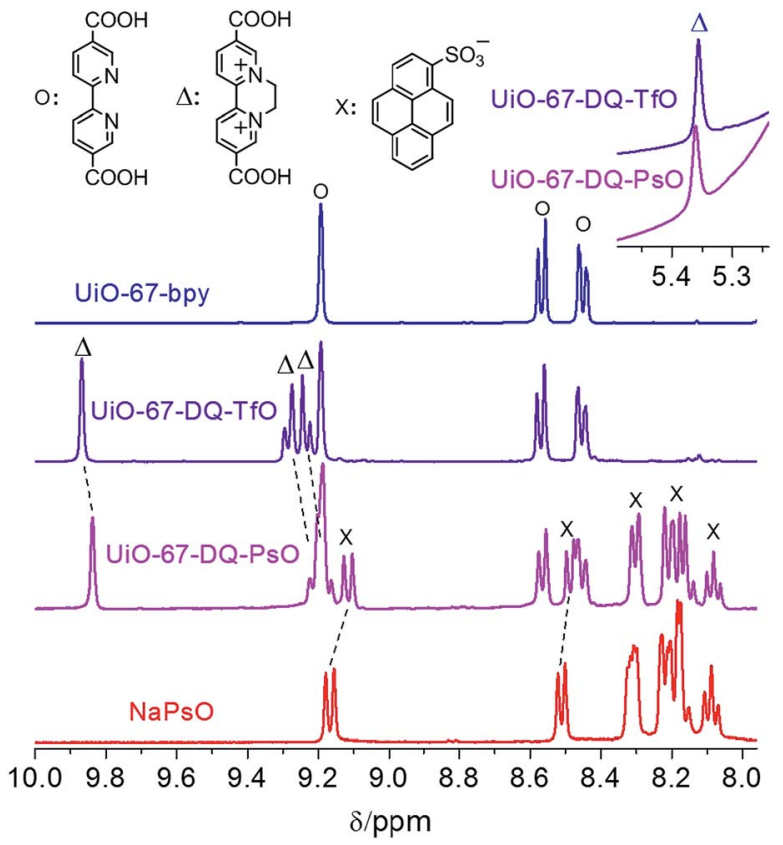

Fig. $1{ }^{1} \mathrm{H}$ NMR spectra of the solutions obtained by digesting UiO-67bpy, UiO-67-DQ-TfO, UiO-67-DQ-PsO and $\mathrm{NaPsO}$ with $\mathrm{HF}($ aq. $) / \mathrm{d}_{6}$ DMSO. The dashed lines stress the shifts of some peaks.

toward a high field after anion exchange, while the bpy peaks remain unshifted. On the other hand, some $\mathrm{PsO}^{-}$peaks for UiO67-DQ-PsO are also obviously shifted compared with those for NaPsO.

The phenomena are indicative of electronic interactions between $\mathrm{PsO}^{-}$and $\mathrm{DQ}^{-}$in solution.

Powder X-ray diffraction (PXRD) profiles of UiO-67-DQ-TfO and UiO-67-DQ-PsO are in good agreement with that of UiO-67bpy (Fig. 2a), confirming that the crystalline framework is essentially retained after postsynthetic $\mathrm{N}$-alkylation and anion exchange. The peak at $2 \theta=9.4^{\circ}$ (index [220]) increases in relative intensity on going from UiO-67-bpy to UiO-67-DQ-TfO and to UiO-67-DQ-PsO. This is consistent with the general phenomenon that the [220] peaks of UiO-66/67 MOFs (at 12.0 for UiO-66) decrease in intensity and even disappear upon guest removal. ${ }^{17}$ XRD simulation for UiO-66/67 reveals that the intensity of the peak is sensitive to the presence/absence of guest species in the pores of the MOFs, so the peak is mainly related to X-ray scattering of the guest. This justifies the change
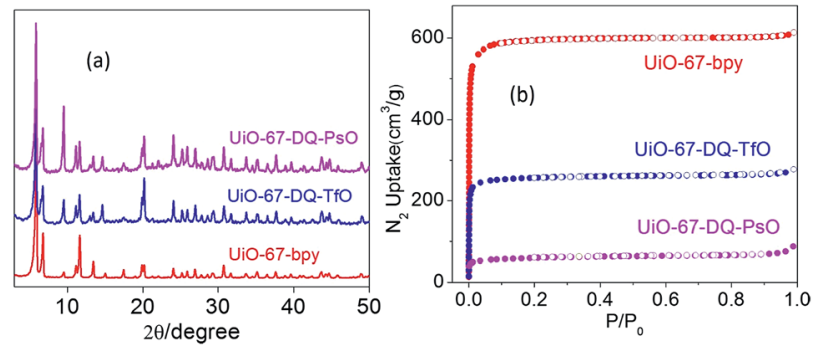

Fig. 2 (a) PXRD patterns and (b) $\mathrm{N}_{2}$ adsorption/desorption isotherms of UiO-67-bpy, UiO-67-DQ-TfO and UiO-67-DQ-PsO. of the peak upon encapsulation of large anions. The XRD profile of UiO-67-DQ-PsO shows a small background hump between 20 and $30^{\circ}(2 \theta)$, which could be tentatively ascribed to a slight structural degradation during anion exchange. The MOFs show a type-I nitrogen adsorption/desorption isotherm characteristic of microporosity (Fig. 2b). The Brunauer-Emmet-Teller (BET) surface area $\left(977 \mathrm{~m}^{2} \mathrm{~g}^{-1}\right)$ and the total pore volume $\left(0.429 \mathrm{~cm}^{3}\right.$ $\mathrm{g}^{-1}$ ) of UiO-67-DQ-TfO are significantly lower than the corresponding values $\left(2047 \mathrm{~m}^{2} \mathrm{~g}^{-1}\right.$ and $\left.0.949 \mathrm{~cm}^{3} \mathrm{~g}^{-1}\right)$ of UiO-67-bpy, owing to the occupation of the pores by the ethylene group and the $\mathrm{TfO}^{-}$anions. The values for UiO-67-DQ-PsO are further decreased $\left(220 \mathrm{~m}^{2} \mathrm{~g}^{-1}\right.$ and $\left.0.136 \mathrm{~cm}^{3} \mathrm{~g}^{-1}\right)$ because of the bulkiness of the $\mathrm{PsO}^{-}$anion. The MOFs show good thermal stability and retain the structural integrity after heating at $300{ }^{\circ} \mathrm{C}$ (UiO-67-DQ-TfO) and $200{ }^{\circ} \mathrm{C}$ (UiO-67-DQ-PsO), according to thermogravimetric and PXRD data (Fig. S3 $\dagger$ ).

\section{Response of UiO-67-DQ-TfO to amines}

The MOF quickly changes from white to orange or red upon exposure to vapors of alkylamines such as butylamine, diethylamine, triethylamine and piperidine (Fig. 3a). By contrast, aniline vapor leads to a very faint color change only after prolonged exposure. Various non-amine VOCs (volatile organic compounds), including aliphatic/aromatic hydrocarbons, halohydrocarbons, alcohols, nitriles, amides, ketones, ethers, esters, and pyridines (only a few are shown in Fig. 3a), cannot cause any detectable color change. The selective chromogenic response to alkylamines is related to the appearance of the strong visible-light absorption band in the solid-state spectra of the amine-treated MOFs (Fig. 3c and $\mathrm{S} 4 \dagger$ ). The optical properties are reflective of CT complex formation between amines and the framework. ${ }^{15 c, 18}$ The precursor MOF UiO-67-bpy shows a negative color response to amines, so it is the $\mathrm{DQ}^{2+}$ chromophore that interacts with amine molecules. The deep colored UiO-67-DQ-TfO samples after amine treatment rapidly fade to their original color if treated with gaseous or aqueous $\mathrm{HCl}$ (Fig. S5a†), so it is the amine group that is responsible for the CT interaction. The fading also occurs when washed with water or just kept in open air. To our delight, the UiO-67-type framework remains intact during the colorationdecoloration cycle, as confirmed by PXRD (Fig. S5b $†$ ).

The high-contrast, selective and reversible chromogenic properties imply potential applications for visual amine detection, food spoilage monitoring and invisible ink. For naked-eye and portable on-site detection, the test paper prepared by dipcoating filter paper with UiO-67-DQ-TfO dispersion shows an immediate color change when exposed to alkylamine vapors or solutions (Fig. S6†). Preliminary experiments indicate that the test paper can detect alkylamine vapors at a low concentration of $5 \mathrm{ppm}$, which is lower than the detection limits of most previous colorimetric sensors for alkylamine vapors (Table S1 $\dagger$ ).

UiO-67-DQ-TfO fluoresces in the solid state and aqueous dispersion. The emission intensity is significantly reduced after being treated with alkylamines (Fig. $3 \mathrm{~b}$ and $\mathrm{c}$ ). Consistent with the selective chromogenic properties, the aniline vapor or solution shows a much weaker quenching effect, and nonamine VOCs do not influence the emission. Considering that 

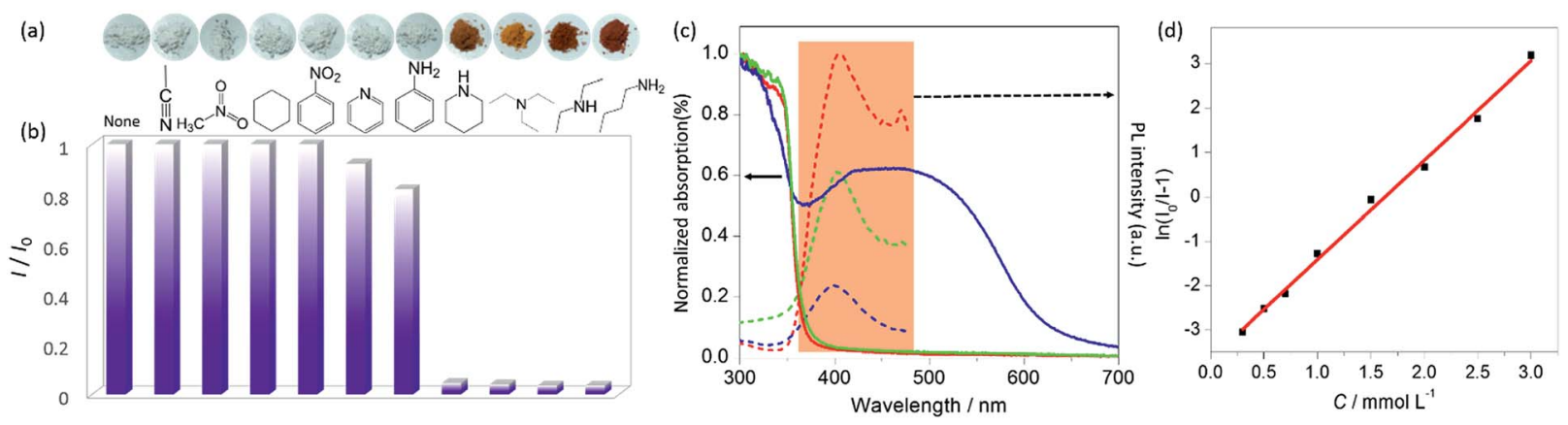

Fig. 3 (a) Photographs of UiO-67-DQ-TfO after being fumed with amines for few seconds. The negative response to some non-amine VOCs is also shown for comparison. (b) Relative fluorescence intensity (at $423 \mathrm{~nm}$ ) of UiO-67-DQ-TfO/ $\mathrm{H}_{2} \mathrm{O}$ dispersions in response to various VOCs (3 $\mathrm{mM}$ ) in water. (c) Solid-state absorption (solid line) and emission spectra (dash line) of UiO-67-DQ-TfO before (red) and after exposure to butylamine (blue) and aniline (green). (d) The variation of $\ln \left(I_{0} / /-1\right)$ upon incremental addition of butylamine to a UiO-67-DQ-TfO/ $\mathrm{H}_{2} \mathrm{O}$ dispersion.

the emission band overlaps with the CT absorption band of the amine-treated MOF (Fig. 3c), the quenching effect could be due to Förster resonance energy transfer (FRET) from the framework to the non-emissive CT complex. To evaluate the performance of UiO-67-DQ-TfO for quantitative determination of alkylamines, the emission spectra of the MOF dispersed in water were monitored while butylamine (BA) was incrementally added. As expected, increasing the BA concentration leads to a gradual decrease in the emission intensity (Fig. S7 $\dagger$ ). The plot of $I_{0} / I$ versus $\mathrm{BA}$ concentration $(C)$ can be fitted to the exponential function $I_{0} / I=A \mathrm{e}^{K C}+1$ (Fig. $3 \mathrm{~d}$ ), where $I$ and $I_{0}$ are the emission intensities at $423 \mathrm{~nm}$ in the presence and absence of BA, respectively. The limit of detection (LOD) was estimated to be $1.7 \times 10^{-4} \mathrm{M}$ by applying the $3 \sigma / S$ convention $(\sigma$ is the standard deviation of the blank measurements, and $S$ is the slope of the $I-C$ plot at a low concentration).

\section{Fluorescence turn-on detection using UiO-67-DQ-PsO}

The fluorescence spectrum of UiO-67-DQ-PsO dispersed in water is compared with those of UiO-67-DQ-TfO and NaPsO in Fig. 4. The $\mathrm{PsO}^{-}$solution shows strong emission bands at 376 , 395 and $415 \mathrm{~nm}\left(\lambda_{\mathrm{ex}}=346 \mathrm{~nm}\right)$, while the emission of UiO-67DQ-PsO is very weak, even much weaker than that of UiO-67DQ-TfO. It is clear that encapsulation of $\mathrm{PsO}^{-}$into the MOF efficiently quenches not only the emission from the framework but also that from the organic anion itself. Therefore, UiO-67DQ-PsO provides an excellent OFF state for potential turn-on sensing. When BA $(10 \mu \mathrm{M})$ was added to UiO-67-DQ-PsO, the $\mathrm{PsO}^{-}$emission was triggered, so the turn-on sensing was achieved. The enhancement ratio $\left(I / I_{0}\right)$ at $395 \mathrm{~nm}$ is as high as 25 , suggesting a high signal-to-background ratio. The fluorescence turn-on is clearly visible to the naked eye under a UV lamp (254 nm) (Fig. 4, inset).

To evaluate the sensitivity of the turn-on detection system for amine, fluorescence titrations were performed by stepwise addition of BA to the aqueous dispersion of UiO-67-DQ (Fig. 5a). An ultratrace amount of $\mathrm{BA}(2 \mathrm{nM})$ causes a high enhancement ratio of 4.3, and the ratio increases linearly with $\log C_{\mathrm{BA}}$ in the range of $10^{-9}$ to $10^{-5} \mathrm{M}\left(I / I_{0}=5.42 \log C_{\mathrm{BA}}+52.29\right.$, Fig. $\left.5 \mathrm{~b}\right)$. The LOD is estimated to be $1.7 \times 10^{-10} \mathrm{M}$. This implies that UiO-67-DQ-PsO can be used as an ultrasensitive luminescent sensor for quantitative detection of alkylamines in the micro- to subnanomolar range. The LOD value is lower by six orders of magnitude compared to that of the turn-off detection using UiO-67-DQ-TfO, emphasizing the superiority of the IDA method.

The alkylamine-responsive fluorescence turn-on is also highly selective. Negligible changes of emissions were observed when the aqueous dispersion of UiO-67-DQ-PsO was treated with aniline and a variety of other organic compounds (Fig. 6a). To determine the interference of excessive aniline with alkylamine sensing, the emission of the UiO-67-DQ-PsO suspension was monitored upon successive addition of BA and incremental amounts of aniline. We found that the emission is only responsive to BA addition and nearly independent of aniline addition, even a large excess of aniline causing no significant change (Fig. 6b). The interference rate measured using $\left(I^{\prime}-I\right) /(I$ $\left.-I_{0}\right) \times 100 \%$ is only $1.0 \%$ for 1000 equivalents of aniline $\left(I^{\prime}\right.$ is the intensity in the copresence of BA and aniline). The trivially low interference rates suggest that the turn-on system has

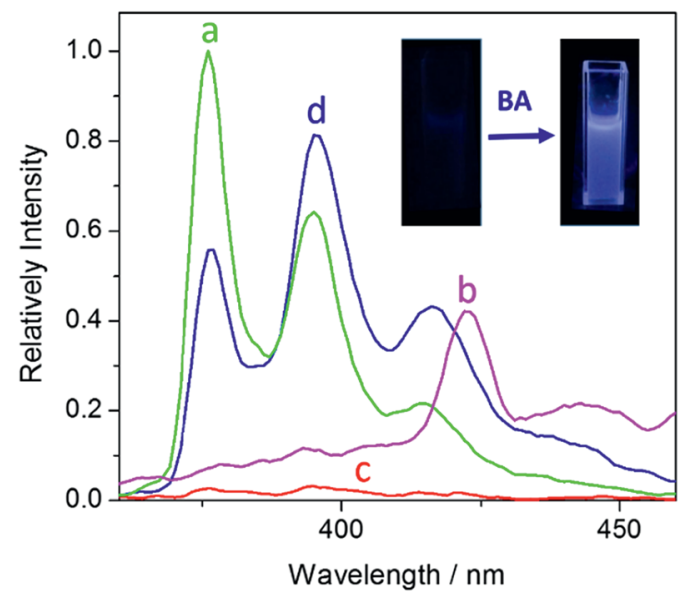

Fig. 4 Emission spectra of $\mathrm{NaPsO}(\mathrm{a})$, UiO-67-DQ-TfO (b), and UiO67-DQ-PsO ((c) original; (d) after addition of butylamine (BA)) dispersed in water. Inset: the visual change under UV light (254 nm) upon addition of BA. 

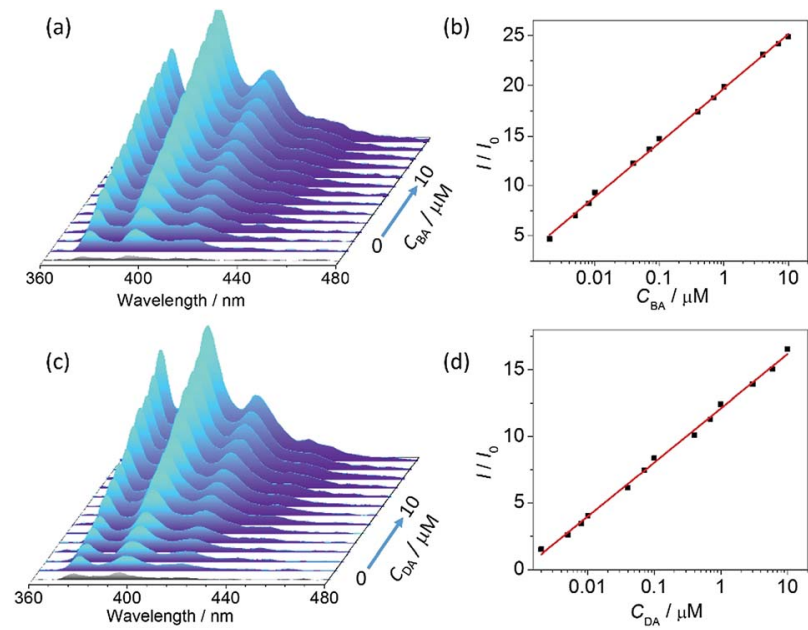

Fig. 5 Variation of the emission spectrum of UiO-67-DQ-PsO dispersed in water upon incremental addition of butylamine (BA, a) and dopamine (DA, c) and the corresponding plots ( $b$ and $d$ ) of $I / I_{0}$ at $395 \mathrm{~nm}$ versus the amine concentration.
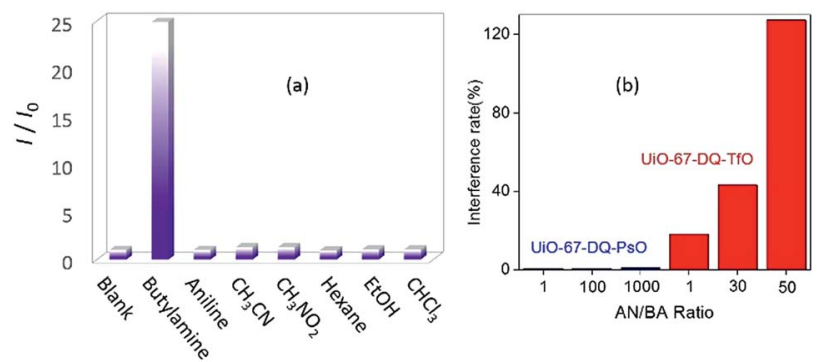

Fig. 6 (a) Relative emission intensity of aqueous $\mathrm{UiO}-67-\mathrm{DQ}-\mathrm{PsO}$ dispersions in response to various organic compounds. (b) The interference rate of different amounts of aniline (AN) for detection of butylamine (BA) using UiO-67-DQ-PsO $\left(C_{\mathrm{BA}}=4 \times 10^{-7} \mathrm{M}\right)$ and UiO$67-D Q-T f O\left(C_{B A}=1 \times 10^{-3} \mathrm{M}\right)$.

excellent anti-interference capability. For comparison, the turnoff detection of BA using UiO-67-DQ-TfO is strongly interfered with by aniline (Fig. 6b), the interference rate being $18 \%$ at 1 equivalent of aniline and increasing rapidly to $127 \%$ at 50 equivalents. The results show that the turn-on IDA using UiO67-DQ-PsO not only dramatically enhances the sensitivity but also significantly improves the anti-jamming performance.

Dopamine (DA) is an important neurotransmitter and medication. Abnormal concentrations of DA have been correlated with various diseases in the mammalian central nervous system, so accurate determination of DA is important for disease diagnosis and monitoring. ${ }^{19}$ To further demonstrate the fluorescence turn-on system, UiO-67-DQ-PsO was tested as a sensor for determination of DA in water. The results obtained with DA are similar to those with BA (Fig. 5c and d). The enhancement ratio is linear with $\log C_{\mathrm{DA}}$, and the LOD is $3.4 \times$ $10^{-10} \mathrm{M}$. The subnanomolar LOD makes UiO-67-DQ-PsO stands out in sensitivity not only among the few MOFs with available LODs $^{13 c, d, 20}$ but also among materials of various classes ${ }^{21}$ reported as luminescence sensors for DA (Table S1†).
Furthermore, from the economic and environmental viewpoints, it is highly desired that a sensory material can be reused through easy regeneration procedures. However, the reusability of MOF-based sensors is often limited by their poor stability against solvents (especially water) and analytes. To test the recyclability of UiO-67-DQ-PsO for DA detection, the used solid was isolated from the suspension by centrifugation, washed with water to remove adsorbed DA, dried and then reused for the next run of detection. As shown in Fig. 7a, the OFF state is recovered in the used MOF after the simple treatments, and the ON state evoked by DA shows no significant degradation in emission intensity compared with that of the fresh sample. Therefore, the sensor is recyclable after simple separation and washing. The reusability is endowed by the high structural stability of the ionic MOF against water and amines, which is evidenced by the PXRD measurements before and after the recycle tests (Fig. 7b). The facile recoverability and recyclability are some of the advantages of the IDA within a solid porous matrix. The homogeneous nature of traditional IDA processes complicates removal and recovery of receptors/indicators from solutions. UiO-67-DQ-PsO is a two-in-one sensor combining both the receptor and indicator in one stable solid, facilitating recovery. It is notable that for the sake of electroneutrality, the anionic indicator $\left(\mathrm{PsO}^{-}\right)$would not leave the cationic framework during amine-sensing.

\section{Mechanism}

To probe the interactions underlying the IDA, the UV-vis spectra of UiO-67-DQ-PsO its precursors (UiO-67-DQ-TfO and NaPsO) (Fig. 8) are compared. UiO-67-DQ-PsO shows a shoulder visiblelight absorption that is absent in the precursors, consistent with the color change (Fig. S8 $\dagger$ ). The differences indicate groundstate PsO-DQ CT complexation. As observed for amine-DQ CT complexation in UiO-67-DQ-TfO, the PsO-DQ CT complexation serves to quench the emission of $\mathrm{PsO}^{-}$and the framework, leading to the OFF state of UiO-67-DQ-PsO. The PsO-DQ interactions also occur in solution: as marked in Fig. 1, some $\mathrm{DQ}^{2+}$ and $\mathrm{PsO}^{-}$NMR peaks for the digested UiO-67-DQ-PsO are shifted compared with the corresponding peaks for the precursors. After being treated with BA, both UiO-67-DQ-PsO and UiO-67-DQ-TfO turn red brown and show significantly
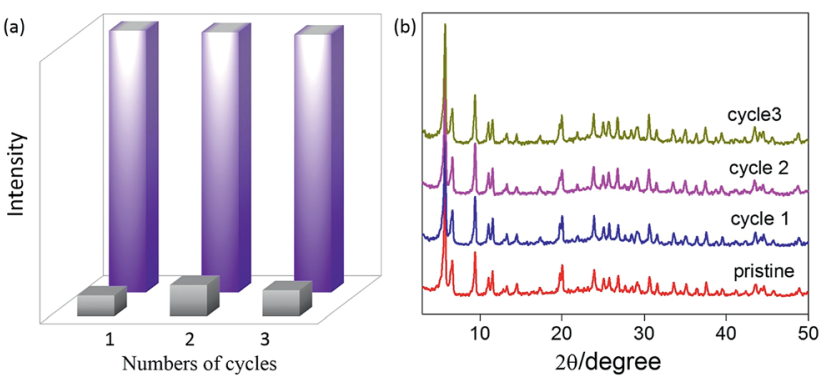

Fig. 7 (a) Reproducibility of the fluorescence off (gray) and on (violet) states of $\mathrm{UiO}-67-\mathrm{DQ}-\mathrm{PsO}$ during three cycles of dopamine sensing (0.01 mM). (b) PXRD patterns of UiO-67-DQ-PsO before and after the cycles. 


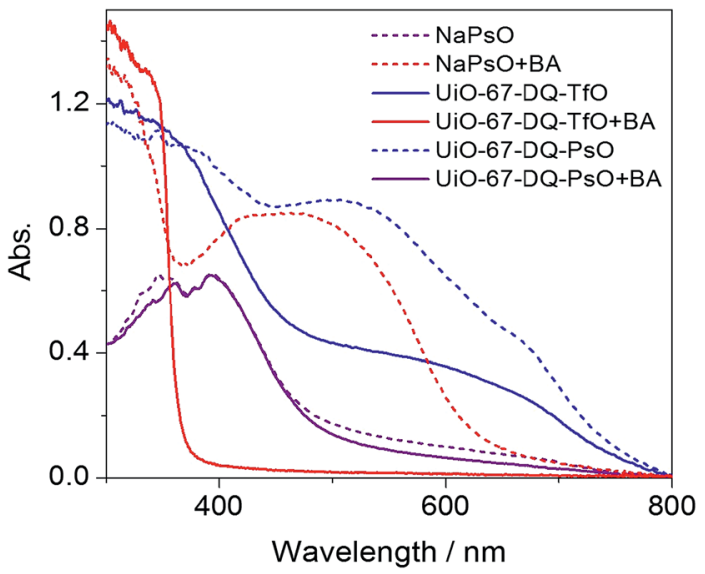

Fig. 8 UV-vis spectra of NaPsO, UiO-67-DQ-TfO and UiO-67-DQ$\mathrm{PsO}$ before (solid lines) and after (dashed lines) exposure to butylamine (BA) vapor.

increased visible-light absorbance, suggesting the formation of CT complexes between the amine and $\mathrm{DQ}^{2+}$ in UiO-67-DQPsO. CT interactions between the amine and $\mathrm{PsO}^{-}$can be precluded because NaPsO shows no color and spectral response to amines. The amine-DQ CT complexation serves to separate $\mathrm{PsO}^{-}$from $\mathrm{DQ}^{2+}$ (but $\mathrm{PsO}^{-}$still remains in the $\mathrm{MOF}$ ), and the fluorescence of the indicator is turned on, completing the IDA mechanism. ${ }^{10 a}$ The high sensitivity implies that the CT interaction of $\mathrm{DQ}^{2+}$ with alkylamine is stronger than that with $\mathrm{PsO}^{-}$in UiO-67-DQ-PsO. Consistent with the mechanism, the excellent anti-interference capability of the alkylaminesensing system toward aniline can be well justified. Aniline can form weak CT complexes with $\mathrm{DQ}^{2+}$ in UiO-67-DQ-TfO, interfering with alkylamine detection. However, the amino group of aniline has weaker Lewis basicity (electron-donor strength) and polarity. It can be assumed that the interactions between aniline and $\mathrm{DQ}^{2+}$ are not sufficiently strong to effectively interfere with the CT complexes between $\mathrm{PsO}^{-}$and $\mathrm{DQ}^{2+}$, so aniline cannot induce indicator displacement and does not interfere in the IDA of alkylamines.

The mechanism is also supported by some results obtained with different MOF-indicator systems. The mixed-anion MOF obtained by incomplete anion exchange (UiO-67-DQ-PsO-TfO with $\mathrm{PsO} / \mathrm{TfO}=2$, see above) has been tested for BA detection (Fig. S9†). No significant fluorescence turn-on was observed at BA concentrations below $1 \mu \mathrm{M}$. The LOD is $1.5 \times 10^{-5} \mathrm{M}$, so the detection sensitivity of the mixed-anion MOF is much lower than that of the completely exchanged MOF, which can be justified as follows. In the mixed-anion MOF, not all of the $\mathrm{DQ}^{2+}$ sites form CT complexes with $\mathrm{PsO}^{-}$. The "free" receptor sites that do not form complexes with the anionic indicators should have priority in interacting with incoming amine molecules. Therefore, the amine cannot separate $\mathrm{PsO}^{-}$from $\mathrm{DQ}^{2+}$ and the fluorescence of $\mathrm{PsO}^{-}$is not turned on until the "free" $\mathrm{DQ}^{2+}$ sites are used up at a higher amine concentration. Similar phenomena have been observed in our initial studies using 2-hydroxy-1,3,6-pyrenetrisulfonate $\left(\mathrm{HPTS}^{3-}\right)$ as the fluorescent indicator. UiO-67-DQ-HPTS, prepared by complete anion exchange of UiO-67-DQ-TfO with $\mathrm{Na}_{3}$ HPTS, also shows a fluorescence turn-on response to alkylamine (Fig. S10 $\dagger$ ), but the sensitivity is also much lower compared with that of UiO-67-DQ-PsO: as shown in Fig. S11, $\dagger$ the addition of $10 \mu \mathrm{M}$ BA to the UiO-67-DQ-HPTS dispersion causes an enhancement ratio of 1.4 , which is much lower than that (25) for UiO-67-DQ-PsO under identical conditions. The low sensitivity of UiO-67-DQ-HPTS can be explained as follows. The charge balance dictates that the amount of the trianionic indicator in the MOF is less than that of the dicationic receptor site, and therefore the "free" $\mathrm{DQ}^{2+}$ sites delay the indicator displacement. The adverse effects of incomplete anion exchange and a higher-charged indicator provides corroborative evidence for the IDA mechanism.

To gain clues as to the possible effects of the confined space in the MOF on the IDA, the interactions of $\mathrm{DQ}^{2+}$ with $\mathrm{PsO}^{-}$and amine in an unconfined environment (i.e., in solution) were probed by monitoring the emission of an NaPsO solution (0.8 mM, equivalent to the amount of UiO-67-DQ-PsO used for sensing experiments) upon successive addition of $\mathrm{DQ}^{2+}$ and BA (Fig. S12 $\dagger$ ). The emission can be quenched by $\mathrm{DQ}^{2+}$, but the quenching efficiency is only $37 \%$ at $C_{\mathrm{PsO}}: C_{\mathrm{DQ}}=1: 0.5$, which corresponds to the ratio in UiO-67-DQ-PsO. For comparison, the $\mathrm{PsO}^{-}$emission is almost completely quenched in UiO-67DQ-PsO. This suggests that the PsO-DQ interaction in solution is dramatically weakened by solvation and cannot afford a good OFF state for IDA. In addition, the addition of excessive BA hardly influenced the emission of the solution mixture of $\mathrm{PsO}^{-}$and $\mathrm{DQ}^{2+}$, suggesting that the IDA cannot be performed using the $\mathrm{DQ}^{2+}-\mathrm{PsO}^{-}$ensemble in homogeneous solutions. To gain further insight, similar studies were performed using aqueous $\left[\mathrm{Me}_{2} \mathrm{DQdc}\right]^{2+}$ (dimethyl diquat-5,5'-dicarboxylate, the ester of the very linker for UiO-67-DQ) (Fig. S13†). We chose the ester instead of the carboxylic acid $\left(\mathrm{H}_{2}\right.$ DQdc) because the acid-base reaction with amine would influence the interactions between bipyridinium and amine. Although the quenching efficiency (53\%) of $\left[\mathrm{Me}_{2} \mathrm{DQdc}\right]^{2+}$ at $C_{\mathrm{PsO}}: C_{\mathrm{Me}_{2} \mathrm{DQdc}}$ $=1: 0.5$ is higher than that $(37 \%)$ of $\mathrm{DQ}^{2+}$, it is still far from the complete quenching observed for UiO-67-DQ-PsO. The addition of $0.8 \mathrm{mM}$ BA (BA : $\mathrm{Me}_{2} \mathrm{DQdc}$ molar ratio $=2: 1$ ) to the $\left[\mathrm{Me}_{2} \mathrm{DQdc}\right]^{2+}-\mathrm{PsO}^{-}$mixture enhances the emission by $30 \%$, indicating a weak indicator displacement effect. However, the enhancement is much lower than that for the UiO-67-DQ-PsO dispersion, where $0.01 \mathrm{mM}$ BA enhances the emission by as much as 24 times (vide supra). That is, the turn-on IDA within the MOF is much more efficient than that in solution. We propose that the confined space provided by the MOF should significantly enhance both receptorindicator and receptor-analyte interactions. Therefore, integrating a receptor-indicator ensemble within a MOF can lead to efficient and sensitive IDA that is insensitive or impossible without space confinement. By the way, the different behaviors between $\mathrm{DQ}^{2+}$ and $\left[\mathrm{Me}_{2} \mathrm{DQdc}\right]^{2+}$ could be due to the electron-withdrawing nature of the carboxylate groups, which makes the bipyridinium moiety more electrondeficient to the advantage of CT interactions with both $\mathrm{PsO}^{-}$ and amine. 


\section{Conclusions}

In summary, we proposed and demonstrated the IDA-in-MOF approach for the design of fluorescence turn-on sensors. The MOF integrated with electron-deficient receptor sites and an electron-rich fluorescent indicator can efficiently sense alkylamines in water because of indicator displacement through competing CT interactions of the receptor with analytes/ indicators. We demonstrated that implementing IDA within the MOF affords several appreciable benefits. The turn-on assay shows dramatically enhanced sensitivity and anti-interference capability compared to the turn-off detection with the indicator-free precursor MOF. The integration of the receptor and indicator into the stable porous solid facilitates the regeneration and recyclability of the IDA ensemble. Furthermore, a strong and positive space-confinement effect was demonstrated. The confined space provided by the MOF can regulate the competing supramolecular interactions for an efficient IDA, which may be inefficient and even impossible in solution. Considering the great diversity and modifiability of MOFs and the various interactions applicable to IDA, the IDAin-MOF approach should be of general interest for the rational design of superior fluorescence turn-on sensors for various analytes. It can also be extended to other porous materials such as porous organic frameworks. This work also has important implications for space-confined supramolecular chemistry in porous solids.

\section{Experimental section}

\section{Synthesis of UiO-67-DQ-TfO}

UiO-67-bpy and EDT were synthesized according to literature methods. $^{22}$ EDT $(750 \mu \mathrm{L})$ was added to UiO-67-bpy (100 mg, $\sim 0.046 \mathrm{mmol})$ in $\mathrm{CHCl}_{3}(2.5 \mathrm{~mL})$. After stirring for $2.5 \mathrm{~h}$ at room temperature, the solid was harvested by filtration, washed with ethanol, and then dried in air.

\section{Preparation of UiO-67-DQ-PsO}

A freshly prepared sample (100 mg) of UiO-67-DQ-TfO was stirred in the aqueous solution $(75 \mathrm{~mL})$ of NaPsO $(200 \mathrm{mg})$ for $5 \mathrm{~h}$. The resulting gray-green solid was isolated, washed with water several times to remove any $\mathrm{NaPsO}$ and then dried at room temperature.

\section{Luminescence sensing measurements}

UiO-67-DQ-TfO or UiO-67-DQ-PsO (6.0 mg) was dispersed in distilled water $(10 \mathrm{~mL})$ and sonicated for $30 \mathrm{~min}$ to form a stable milk white suspension. After recording the fluorescence using $2.5 \mathrm{~mL}$ of the dispersion, an aqueous solution containing the analyte with a given concentration was added to the cuvette and stirred for $30 \mathrm{~min}$, and then fluorescence was measured.

\section{Preparation of test paper}

The filter paper was dipped into a dispersion of UiO-67-DQ-TfO in $\mathrm{EtOH}$ (10 $\mathrm{mg} \mathrm{mL}^{-1}$, uniformly dispersed by ultrasonication) for a few seconds and then left to dry at room temperature. The dip-coating and drying procedure was repeated three times.

\section{Conflicts of interest}

There are no conflicts to declare.

\section{Acknowledgements}

This work is supported by the National Natural Science Foundation of China (Grant No. 21773070 and 21471057).

\section{Notes and references}

1 (a) H. Furukawa, K. E. Cordova, M. O'Keeffe and O. M. Yaghi, Science, 2013, 341, 1230444; (b) Y. Zhang, S. Yuan, G. Day, X. Wang, X. Yang and H.-C. Zhou, Coord. Chem. Rev., 2018, 354, 28; (c) W. P. Lustig, S. Mukherjee, N. D. Rudd, A. V. Desai, J. Li and S. K. Ghosh, Chem. Soc. Rev., 2017, 46, 3242.

2 (a) Y. Cui, B. Li, H. He, W. Zhou, B. Chen and G. Qian, Acc. Chem. Res., 2016, 49, 483; (b) R. W. Huang, Y. S. Wei, X. Y. Dong, X. H. Wu, C. X. Du, S. Q. Zang and T. C. W. Mak, Nat. Chem., 2017, 9, 689.

3 (a) A. Karmakar, P. Samanta, A. V. Desai and S. K. Ghosh, Acc. Chem. Res., 2017, 50, 2457; (b) S. M. Cohen, Chem. Rev., 2012, 112, 970.

4 (a) B. Yan, Acc. Chem. Res., 2017, 50, 2789; (b) F. Wang, W. Liu, S. J. Teat, F. Xu, H. Wang, X. Wang, L. An and J. Li, Chem. Commun., 2016, 52, 10249; (c) Z. Ding, J. Tan, G. Feng, Z. Yuan, C. Wu and X. Zhang, Chem. Sci., 2017, 8, 5101.

5 (a) R. Haldar, R. Matsuda, S. Kitagawa, S. J. George and T. K. Maji, Angew. Chem., Int. Ed., 2014, 53, 11772; (b) Y. Takashima, V. M. Martinez, S. Furukawa, M. Kondo, S. Shimomura, H. Uehara, M. Nakahama, K. Sugimoto and S. Kitagawa, Nat. Commun., 2011, 2, 168.

6 (a) Y. Li, S. Zhang and D. Song, Angew. Chem., Int. Ed., 2013, 52, 710; (b) J.-N. Haoand and B. Yan, Chem. Commun., 2015, 51, 7737; (c) B. Zhao, X.-Y. Chen, P. Cheng, D.-Z. Liao, S.-P. Yan and Z.-H. Jiang, J. Am. Chem. Soc., 2004, 126, 15394.

7 V. Martínez-Martínez, S. Furukawa, Y. Takashima, I. López Arbeloa and S. Kitagawa, J. Phys. Chem. C, 2012, 116, 26084. 8 (a) H. Wang, W. P. Lustig and J. Li, Chem. Soc. Rev., 2018, 47, 4729; (b) F. Y. Yi, D. X. Chen, M. K. Wu, L. Han and H. L. Jiang, Chempluschem, 2016, 81, 675; (c) Z. Hu, B. J. Deibert and J. Li, Chem. Soc. Rev., 2014, 43, 5815; (d) R.-B. Lin, S.-Y. Liu, J.-W. Ye, X.-Y. Li and J.-P. Zhang, Adv. Sci., 2016, 3, 1500434; (e) H.-R. Fu, L.-B. Yan, N.-T. Wu, L.-F. Ma and S.-Q. Zang, J. Mater. Chem. A, 2018, 6, 9183; (f) P. Kumar, A. Deep and K.-H. Kim, Trends Anal. Chem., 2015, 73, 39; $(g)$ B. Wang, X.-L. Lv, D. Feng, L.-H. Xie, J. Zhang, M. Li, Y. Xie, J.-R. Li and H.-C. Zhou, J. Am. Chem. Soc., 2016, 138, 6204; (h) S. Wu, Y. Lin, J. Liu, W. Shi, G. Yang and P. Cheng, Adv. Funct. Mater., 2018, 28, 1707169. 
9 (a) S. S. Nagarkar, A. V. Desai and S. K. Ghosh, Chem.-Eur. J., 2015, 21, 9994; (b) Y.-Z. Chenand and H.-L. Jiang, Chem. Mater., 2016, 28, 6698; (c) B. Chen, L. Wang, F. Zapata, G. Qian and E. B. Lobkovsky, J. Am. Chem. Soc., 2008, 130, 6718; (d) N. B. Shustova, A. F. Cozzolino, S. Reineke, M. Baldo and M. Dinca, J. Am. Chem. Soc., 2013, 135, 13326; (e) Y. Guo, X. Feng, T. Han, S. Wang, Z. Lin, Y. Dong and B. Wang, J. Am. Chem. Soc., 2014, 136, 15485; (f) R. N. Das, M. Debnath, A. Gaurav and J. Dash, Chem.Eur. J., 2014, 20, 16688; $(g)$ K. S. Lim, S. Y. Jeong, D. W. Kang, J. H. Song, H. Jo, W. R. Lee, W. J. Phang, D. Moon and C. S. Hong, Chem.-Eur. J., 2017, 23, 4803; (h) M. Zhang, G. Feng, Z. Song, Y.-P. Zhou, H.-Y. Chao, D. Yuan, T. T. Y. Tan, Z. Guo, Z. Hu, B. Z. Tang, B. Liu and D. Zhao, J. Am. Chem. Soc., 2014, 136, 7241; (i) N. B. Shustova, A. F. Cozzolino and M. Dinca, J. Am. Chem. Soc., 2012, 134, 19596; (j) B. Chen, Y. Yang, F. Zapata, G. Lin, G. Qian and E. B. Lobkovsky, Adv. Mater., 2007, 19, 1693; (k) H. Xu, X. Rao, J. Gao, J. Yu, Z. Wang, Z. Dou, Y. Cui, Y. Yang, B. Chen and G. Qian, Chem. Commun., 2012, 48, 7377; (l) D. Yue, D. Zhao, J. Zhang, L. Zhang, K. Jiang, X. Zhang, Y. Cui, Y. Yang, B. Chen and G. Qian, Chem. Commun., 2017, 53, 11221.

10 (a) L. You, D. Zha and E. V. Anslyn, Chem. Rev., 2015, 115, 7840; (b) B. T. Nguyenand and E. V. Anslyn, Coord. Chem. Rev., 2006, 250, 3118; (c) A. Norouzy, Z. Azizi and W. M. Nau, Angew. Chem., Int. Ed., 2015, 54, 792; (d) P. Koutnik, E. G. Shcherbakova, S. Gozem, M. G. Caglayan, T. Minami and P. Anzenbacher Jr, Chem, 2017, 2, 271; (e) Z. Zheng, W.-C. Geng, J. Gao, Y.-Y. Wang, H. Sun and D.-S. Guo, Chem. Sci., 2018, 9, 2087; $(f)$ M. A. Beatty, J. Borges-Gonzalez, N. J. Sinclair, A. T. Pye and F. Hof, J. Am. Chem. Soc., 2018, 140, 3500; $(g)$ B. M. Chapin, P. Metola, S. L. Vankayala, H. L. Woodcock, T. J. Mooibroek, V. M. Lynch, J. D. Larkin and E. V. Anslyn, J. Am. Chem. Soc., 2017, 139, 5568.

11 (a) S. A. Lawrence, Amines: Synthesis, Properties and Applications, Cambridge University Press, Cambridge, 2004; (b) M. Papageorgiou, D. Lambropoulou, C. Morrison, E. Klodzinska, J. Namiesnik and J. Plotka-Wasylka, Trends Anal. Chem., 2018, 98, 128.

12 (a) S.-S. Zhao, J. Yang, Y.-Y. Liu and J.-F. Ma, Inorg. Chem., 2016, 55, 2261; (b) X. Y. Xu, X. Lian, J. N. Hao, C. Zhang and B. Yan, Adv. Mater., 2017, 29, 1702298; (c) J. Chen, F.-Y. Yi, H. Yu, S. Jiao, G. Pang and Z.-M. Sun, Chem. Commun., 2014, 50, 10506; (d) S. Khatua, S. Goswami, S. Biswas, K. Tomar, H. S. Jena and S. Konar, Chem. Mater., 2015, 27, 5349; (e) P. Rouschmeyer, N. Guillou, C. Serre, G. Clavier, C. Martineau, P. Audebert, E. Elkaim, C. Allain and T. Devic, Inorg. Chem., 2017, 56, 8423.

13 (a) P. Mani, A. A. Ojha, V. S. Reddy and S. Mandal, Inorg. Chem., 2017, 56, 6772; (b) S. W. Jaros, J. Sokolnicki, A. Woloszyn, M. Haukka, A. M. Kirillov and P. Smolenski,
J. Mater. Chem. C, 2018, 6, 1670; (c) C. Zhao, Z. Jiang, R. Mu and Y. Li, Talanta, 2016, 159, 365; (d) Y. Cheng, J. Wu, C. Guo, X.-G. Li, B. Ding and Y. Li, J. Mater. Chem. $B, 2017,5,2524$.

14 (a) A. Mallick, B. Garai, M. A. Addicoat, P. S. Petkov, T. Heine and R. Banerjee, Chem. Sci., 2015, 6, 1420; (b) T. Gong, P. Li, Q. Sui, J. Chen, J. Xu and E.-Q. Gao, J. Mater. Chem. A, 2018, 6, 9236; (c) J.-J. Liu, Y.-B. Shan, C.-R. Fan, M.-J. Lin, C.-C. Huang and W.-X. Dai, Inorg. Chem., 2016, 55, 3680; (d) J.-J. Liu, Y.-F. Guan, M.-J. Lin, C.-C. Huang and W.-X. Dai, Cryst. Growth Des., 2015, 15, 5040.

15 (a) Q. Zhang, J. Yu, J. Cai, L. Zhang, Y. Cui, Y. Yang, B. Chen and G. Qian, Chem. Commun., 2015, 51, 14732; (b) L. Xu, Y. Luo, L. Sun, S. Pu, M. Fang, R. X. Yuan and H. B. Du, Dalton Trans., 2016, 45, 8614; (c) N. N. Yang, W. Sun, F. G. Xi, Q. Sui, L. J. Chen and E. Q. Gao, Chem. Commun., 2017, 53, 1747; (d) N. N. Yang, J. J. Fang, Q. Sui and E. Q. Gao, ACS Appl. Mater. Interfaces, 2018, 10, 2735.

16 S. J. Angus-Dunne, L. E. P. Lee Chin, R. C. Burns and G. A. Lawrance, Transition Met. Chem., 2006, 31, 268.

17 (a) C. J. Hafizovic, S. Jakobsen, U. Olsbye, N. Guillou, C. Lamberti, S. Bordiga and K. P. Lillerud, J. Am. Chem. Soc., 2008, 130, 13850; (b) A. Schaate, P. Roy, A. Godt, J. Lippke, F. Waltz, M. Wiebcke and P. Behrens, Chem.Eur. J., 2011, 17, 6643; (c) F.-G. Xi, Y. Yang, H. Liu, H.-F. Yao and E.-Q. Gao, RSC Adv., 2015, 5, 79216.

18 C. J. Bender, Chem. Soc. Rev., 1986, 15, 475.

19 (a) X. Liu, T. Xiao, F. Wu, M.-Y. Shen, M. Zhang, H.-h. Yu and L. Mao, Angew. Chem., Int. Ed., 2017, 56, 11802; (b) A. Pandikumar, G. T. Soon How, T. P. See, F. S. Omar, S. Jayabal, K. Z. Kamali, N. Yusoff, A. Jamil, R. Ramaraj, S. A. John, H. N. Lim and N. M. Huang, RSC Adv., 2014, 4, 63296; (c) M. Sajid, M. K. Nazal, M. Mansha, A. Alsharaa, S. M. S. Jillani and C. Basheer, Trends Anal. Chem., 2016, 76, 15; (d) J.-J. Fang, N.-N. Yang and E.-Q. Gao, Electrochem. Commun., 2018, 89, 32.

20 (a) C. Zhao, Y. Liu and Y. Li, Anal. Sci., 2015, 31, 1035; (b) Q. Zhu, Y. Chen, W. Wang, H. Zhang, C. Ren, H. Chen and X. Chen, Sens. Actuators, B, 2015, 210, 500.

21 (a) Y.-K. Linand and Y.-C. Yeh, Anal. Chem., 2017, 89, 11178; (b) N. Pallikkarathodi Mani, M. Ganiga and J. Cyriac, Analyst, 2018, 143, 1691; (c) D. Bharathi, B. Siddlingeshwar, R. H. Krishna, V. Singh, N. Kottam, D. D. Divakar and A. AbdullahAlkheraif, J. Fluoresc., 2018, 28, 573; (d) C. Sun, F. Yuan, H. Li and X. Wu, Microchim. Acta, 2018, 185, 1; (e) M. I. Halawa, F. Wu, T. H. Fereja, B. Lou and G. Xu, Sens. Actuators, B, 2018, 254, 1017; (f) Y.-S. He, C.-G. Pan, H.-X. Cao, M.-Z. Yue, L. Wang and G.-X. Liang, Sens. Actuators, B, 2018, 265, 371.

22 (a) G. Nickerl, M. Leistner, S. Helten, V. Bon, I. Senkovska and S. Kaskel, Inorg. Chem. Front., 2014, 1, 325; (b) M. Kuroboshi, T. Kondo and H. Tanaka, Heterocycles, 2015, 90, 723 . 